\title{
PERFORMANCE STUDY OF ADHOC ROUTING PROTOCOLS FOR CBR
} TRAFFIC

\author{
Sunil Vyas ${ }^{1}$, Vishal Srivastava ${ }^{2}$ \\ ${ }^{1,2}$ Arya College of Engineering \& IT Kukas, Jaipur, India, fine.sunil85@gmail.com, vishal500371@yahoo.co.in
}

\begin{abstract}
Mobile ad hoc network (MANET) is a dynamic network without fixed infrastructure due to their wireless nature and can be deployed as multi-hop packet networks. The nodes are free to move about and organize themselves into a network. These nodes change position frequently. A Reactive (on-demand) routing strategy is a popular routing category for wireless adhoc routing. The primary objective of this paper is to do comparative study of the performance of routing protocols Destination-Sequenced Distance-Vector (DSDV), Dynamic Source Routing (DSR) and Ad-hoc on demand Distance Vector (AODV) for wireless ad hoc networks in a simulated environment against varying network parameters. The evaluations are done by means of simulations using NS-2 network simulator. The study was done on the basis of performance metrics: throughput, packet delivery function, end-to-end delay, routing overhead and packet lost. Simulation results show that despite in most simulations reactive routing protocols DSR and AODV performed significantly better than proactive routing protocol DSDV for the CBR based traffic.
\end{abstract}

Keywords- component; Mobile Adhoc Network, Routing protocol, DSR, AODV, DSDV

\section{INTRODUCTION}

A Mobile ad hoc network is a group of wireless mobile computers in which nodes collaborate by forwarding packets for each other to allow them to communicate outside the range of direct wireless transmission. MANET is a kind of wireless ad-hoc network and it is a self-configuring network of mobile routers (and associated hosts) connected by wireless links -the union of which forms an arbitrary topology. We are doing a comparative performance investigation for reactive and proactive routing protocols by using different parameters for CBR based traffic. In proactive routing protocols or tabledriven routing protocols each node attempts to maintain consistent, up-to-date routing information from each node to every other node in the network so that when a packet needs to be forwarded, the route is already known and can be immediately used. However, it incurs additional overhead cost due to maintaining up-to-date information and as a result; throughput of the network may be affected but it provides the actual information to the availability of the network. Distance vector (DV) protocol, Destination Sequenced Distance Vector (DSDV) protocol, Wireless Routing protocol Fisheye State Routing (FSR) protocol are the examples of Proactive protocols. Destination-Sequenced Distance-Vector (DSDV) routing protocol is a table-driven algorithm based on the classical Bellman-Ford routing mechanism. Every mobile node in the network maintains a routing table in which all of the possible destinations within the network and the number of hops to each destination are recorded. Whereas in reactive routing technique which is also known as on-demand routing, It takes a different approach of routing which overcomes the disadvantages of proactive routing (D. Kim, J. Garcia and K. Obraczka,2003). In reactive approaches those nodes which require connectivity to the Internet reactively find Internet gateways by means of broadcasting some kind of solicitation within the entire ad hoc network. This approach reduces the overhead of maintaining the route table as that of proactive. The node dynamically checks the route table, and if it does not find an entry for its destination or it finds an outdated entry it performs route discovery to find the path to its destination. The most efficient algorithms, Dynamic Source Routing (DSR) and Adhoc On Demand Distance Vector (AODV) comes under this category. The Dynamic Source Routing protocol (DSR) is a simple and efficient routing protocol designed specifically for use in multi-hop wireless ad hoc networks of mobile nodes. DSR allows the network to be completely self-organizing and self-configuring, without the need for any existing network infrastructure or administration. Dynamic Source Routing, DSR, is a reactive routing protocol that uses source routing to send packets. It uses source routing which means that the source must know the complete hop sequence to the destination (IETF, 2004). Each node maintains a route cache, where all routes it knows are stored. The route discovery process is initiated only if the desired route cannot be found in the route cache. As mentioned before, DSR uses source routing, i.e. the source determines the complete sequence of hops that each packet should traverse. Another advantage of source routing is that it avoids the need for up-to-date routing information in the intermediate nodes through which the packets are forwarded since all necessary routing information is included in the packets. Finally, it avoids routing loops easily because the complete route is determined by a single node instead of 
making the decision hop-by-hop. The Ad Hoc On-demand Distance Vector Routing (AODV) protocol is a reactive unicast routing protocol for mobile ad hoc networks. As a reactive routing protocol, AODV only needs to maintain the routing information about the active paths. In AODV, routing information is maintained in routing tables at nodes. Moreover, AODV adopts the destination sequence number technique used by DSDV in an on-demand way.

\section{RESEARCH METHODOLOGY}

Das, Perkins, and Royer compares the performance of two prominent on demand routing protocols for mobile ad hoc networks - Dynamic Source Routing (DSR) and Ad Hoc OnDemand Distance Vector Routing (AODV). A detailed simulation model with MAC and physical layer models is used to study interlayer interactions and their performance implications (S.K. Sarkar, T.G. Basawaraju and C Puttamadappa, 2008) (C.K. Toh, 2002).

The paper demonstrate that even though DSR and AODV share a similar on demand behavior, the differences in the protocol mechanics can lead to significant performance differentials. We have done performance study for both reactive and proactive protocols by taking different parameters for simulation in NS2.Parameters used for simulation purpose are Node mobility, Network Size, Maximum no. of connections of nodes with source nodes and Data rate solution in order to facilitate maximum It is observed that DSDV have a strong effect on their performance as the mobility of the nodes increases so they may not be suitable for the Ad hoc environments with high mobility circumstance(K. Brown and S. Singh, 1997) (Steve Wisniewski , 2004). The DSR and AODV performed significantly better than DSDV as they use reactive approach, which eliminates the need to periodically flood the network as compared to table driven approach (proactive approach). DSR has overall good performance even when the mobility is high. (Andrea Goldsmith, 2005) (Bing Lin and I. Chlamtac, 2000)

(Wireless_technologies_advantage, 2008). So we can conclude that there is a need for routing protocols explicitly tuned to the characteristics of ad-hoc networks because the performance of the routing protocols depends on the number of parameters like mobility rate, network size, maximum connections to nodes and data rate. It is observed that DSR and AODV outperform DSDV in most of the cases.

\section{PROPOSED WORK}

Ns-2 is a discrete event simulator targeted at networking research. It provides substantial support for simulation of TCP, routing and multicast protocols over wired and wireless networks. It consists of two simulation tools. The network simulator (ns) contains all commonly used IP protocols. The network animator (nam) is use to visualize the simulations. Ns2 fully simulates a layered network from the physical radio transmission channel to high-level applications. Parameters for this environment was set as simulation time for $200 \mathrm{sec}$, environment size for $1000 * 1000$ meter , packet size for 512 bytes , CBR as traffic type and with varying network size and network mobility

\subsection{Performance Metrics}

\subsubsection{Throughput (Kbytes/second)}

It is defined as total number of packets received by the destination. It is a measure of effectiveness of a routing protocol. Finally what matters is the number of packets delivered successfully [6].

\subsubsection{Packet Delivery Fraction (\%)}

It is the ratio of the data packets delivered to the destinations to those generated by the sources. This metric characterizes both the completeness and correctness of the routing protocol. It is a measure of efficiency of the protocol.

\subsubsection{Average End-to-End Delay (seconds)}

It is the average amount of time taken by a packet to go from source to destination.

\subsubsection{Routing overhead (packets)}

It is the total number of routing packets transmitted during the simulation.

\subsubsection{Packets Lost (Packets)}

It is a measure of the number of packets dropped by the routers due to various reasons. The reasons we have considered for evaluation are collisions, time outs, looping, and errors.

\section{SIMULATED RESULT AND OBSERVATION}

We observe from chart 4.1 that source routing protocols AODV and DSR maintain constant throughput regardless of the mobility rate. DSDV on the other hand has difficulties in finding routes when mobility increases. DSDV initially shows throughput of $25.50 \mathrm{Kbytes} / \mathrm{s}$ at pause time of 0 second, but increases to $29.04 \mathrm{Kbytes} / \mathrm{s}$ as the pause time increased to 150 seconds. 


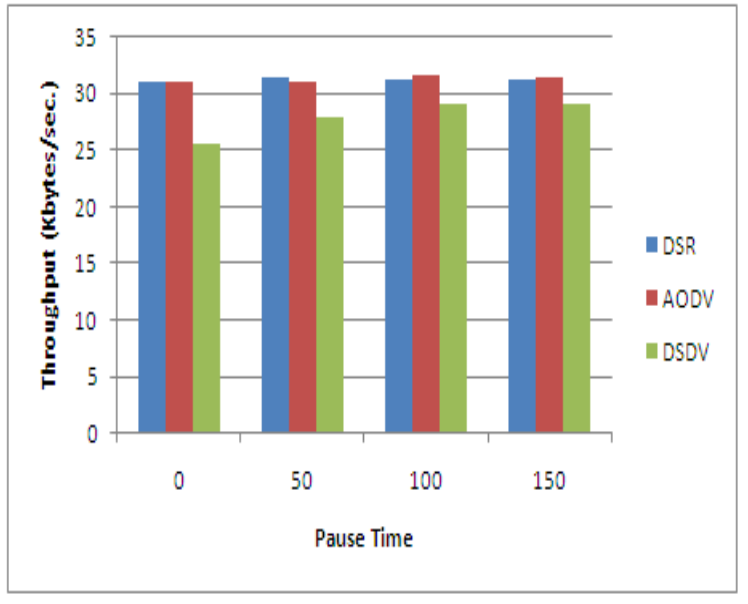

Chart 4.1: Throughput as a function of Node Mobility

All the three protocols DSDV, DSR and AODV deliver a greater percentage of the originated data packets at low node mobility as mentioned below in chart 4.2 (i.e. at large pause time), converging to $99 \%$ delivery of packets when there is no node motion (chart 4.2). DSR and AODV perform particularly well, delivering over $99 \%$ of the data packets regardless of mobility rate. At higher rates of mobility (lower pause times), DSDV does poorly, dropping to a $80 \%$ packet delivery ratio.

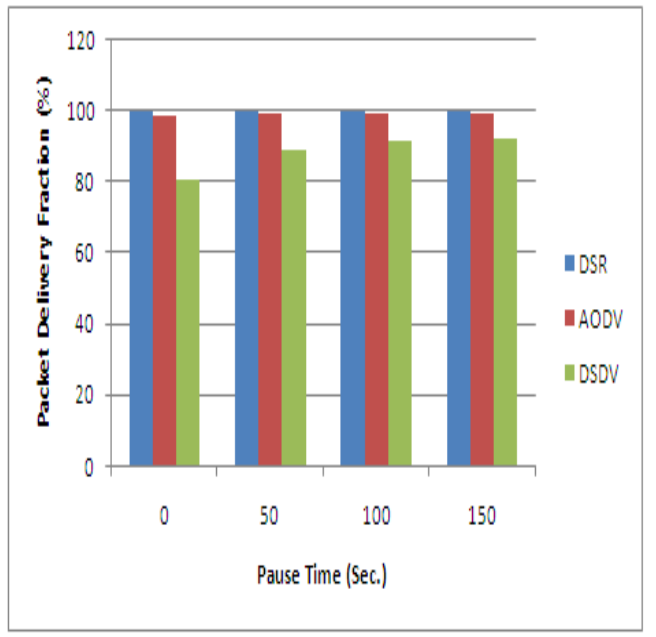

Chart 4.2: Packet Delirery Fraction as a function of node mobility rate (pause time)

From chart 4.3 we observe that the average packet delay increases with mobility for all the three protocols as shown in Figure 4.3. DSDV shows highest delay at pause time of 0 seconds and shortest end-to-end delay of the order of 0.015 seconds when the nodes are in motion because only packets belonging to valid routes at the sending instant get through. The source routing protocols have a longer delay because their route discovery takes more time as every intermediate node tries to extract information before forwarding the reply

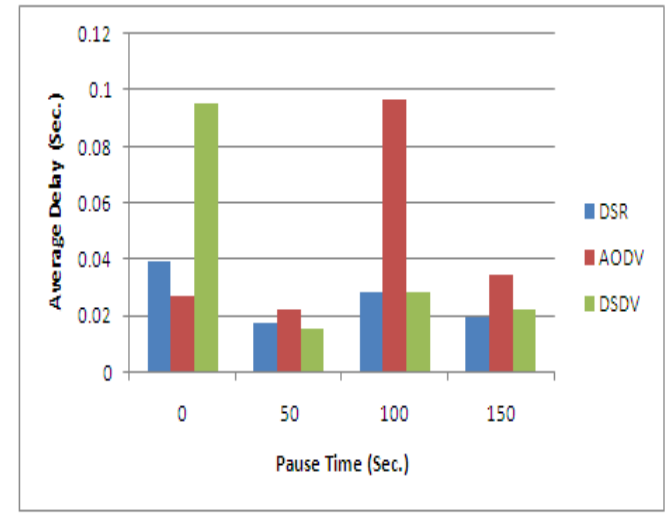

Chart 4.3: Average end-to-end delay as a function of node mobility rate (pause time)

Chart 4.4 shows that routing overhead for source routing protocols decreases as the mobility decreases. Among source routing protocols, AODV shows greater overheads than DSR, transmitting 7963 packets whereas DSR is able to transmit 5803 packets at pause time of 0 second because AODV broadcasts periodic HELLO messages to its neighbors and needs to send control messages more frequently to find and repair routes. DSDV imposes a near about similar overhead transmitting near about 8000 to 9000 packets in the network at all pause times because of the periodic nature of the routing updates.

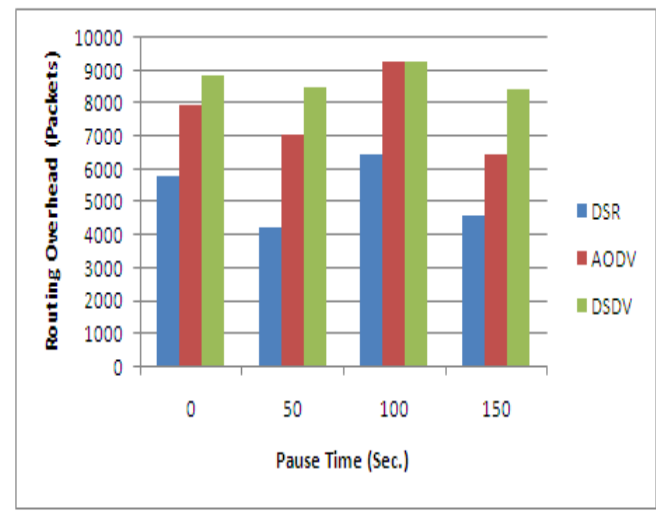

Chart 4.4: Routing overhead as a function of node mobility rate (pause time)

The number of packets lost is quite high initially for DSDV, dropping 296 packets at pause time of 0 second (Chart 4.5) because of high movement of nodes. As pause time of nodes increases, the number of packets loss fall, drops 116 packets at pause time of 150 seconds and it directly affects the number of packets that reach destination. It is clear from here that the performance of DSDV mainly depends upon pause time. For source routing protocols, DSR and AODV, packets lost are quite low and shows negligible packet loss at high mobility. 


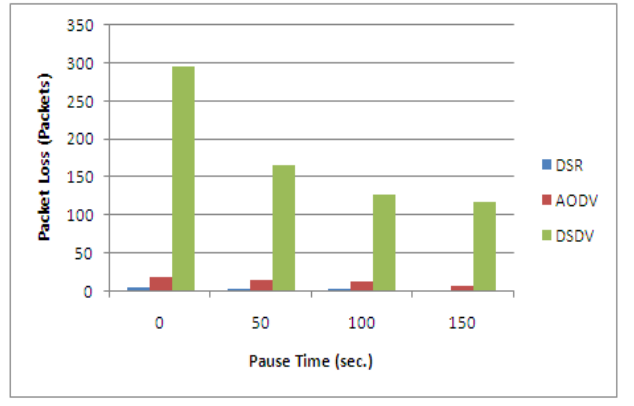

Chart 4.5: Packets Lost as a function of node mobility rate (pause time)

Simulation is conducted for four different size networks of 70 , 100, 120 and 150 wireless nodes, generated for a pause time of 100 seconds. Chart 4.6 shows that none of the three protocols shows significant change on the throughput (Kilobytes/second) with the change in node density. DSDV shows slight variation in the throughput with the change in node density.

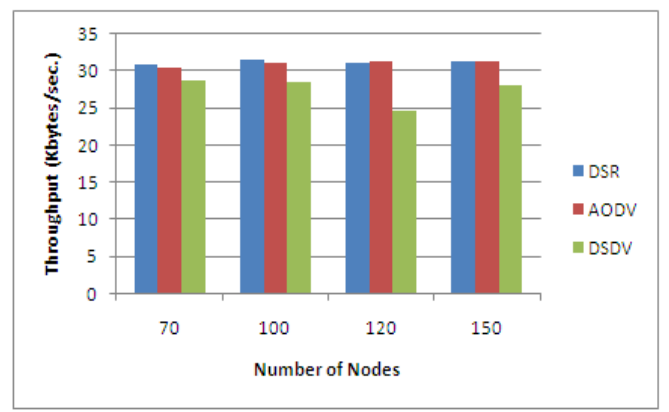

Chart 4.6: Throughput as a function of varying nodes

It is observed that source routing protocols shows significant effect on the packet delivery fraction with the change in network size (Chart 4.7). DSR and AODV shows constant packet delivery ratio above $97 \%$ for any node density due to its source routing nature whereas DSDV shows change in the packet delivery ratio with the change in scalability which is less.

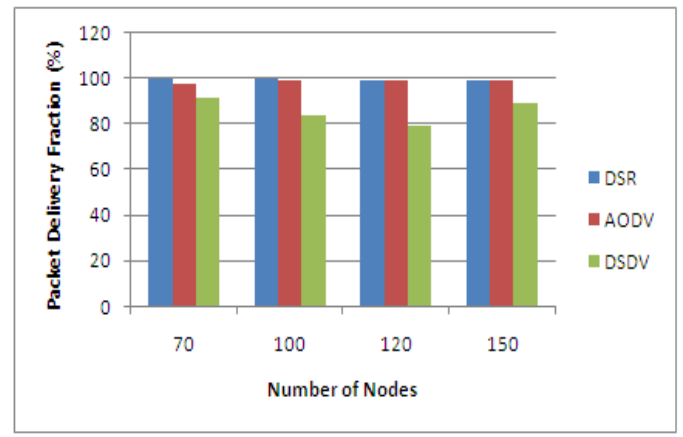

Chart 4.7: Packet delivery fraction as a function of varying nodes
As per study of Chart 4.8 , it is observed that AODV shows the maximum delay of 0.030 seconds in the network of 100 nodes but delay falls to 0.020 seconds when the node density increases to 150 nodes. DSR also behaves with high delay as node density increases as per observations obtained it is found that at node density of 70 nodes the average delay is around 0.025 seconds and at 120 nodes density delay increased to 0.026 seconds which is a slight variation. When density of the nodes increases, route acquisition time also increases considerably. Route Acquisition time is the time required to establish routes when required. Route acquisition time has increased significantly but delay is still decreasing because there is more number of routes available from source to the destination and there are very less route make/break in such situations. Whereas DSDV do not show much change in delay with the change in node density as compared to AODV and DSR protocol.

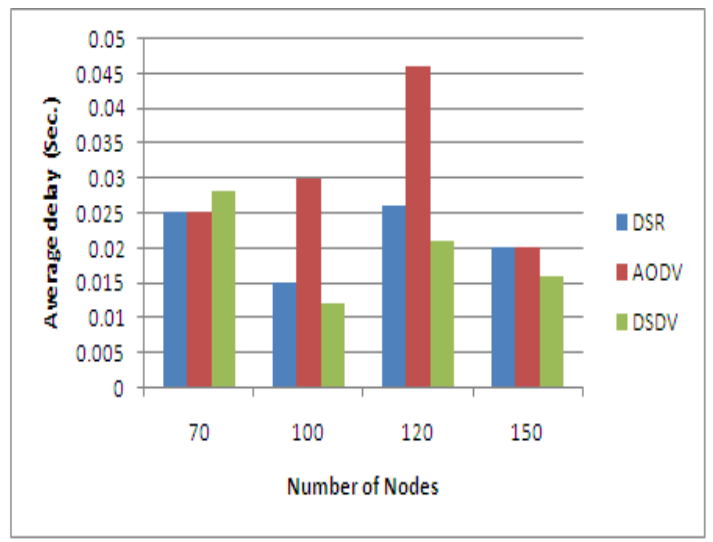

Chart 4.8: Average delay as a function of varying nodes

DSR shows minimum overheads transmitting 5696 packets in the network of 70 nodes but introduces increase in overheads transmitting 6047 packets in the network of 120 nodes as shown in Chart 4.9 and this in turn reduces the efficiency of DSR protocol. Data packet header of DSR carries complete hop by- hop source route to destination, thereby increases overhead with the increase in network size. Another reason for DSR is variable header size due to inclusion of address of intermediate nodes present on route from source to destination. AODV as source routing protocol also shows significant variation in routing overhead as transmission is found 7014 packets at 70 node densities and at 120 nodes routing overhead about 7117 packets for transmission is found and in the case of DSDV, variation in the overheads is found with a margin 7412 packets at 70 nodes and 10734 at 150 nodes due to its proactive approach. 


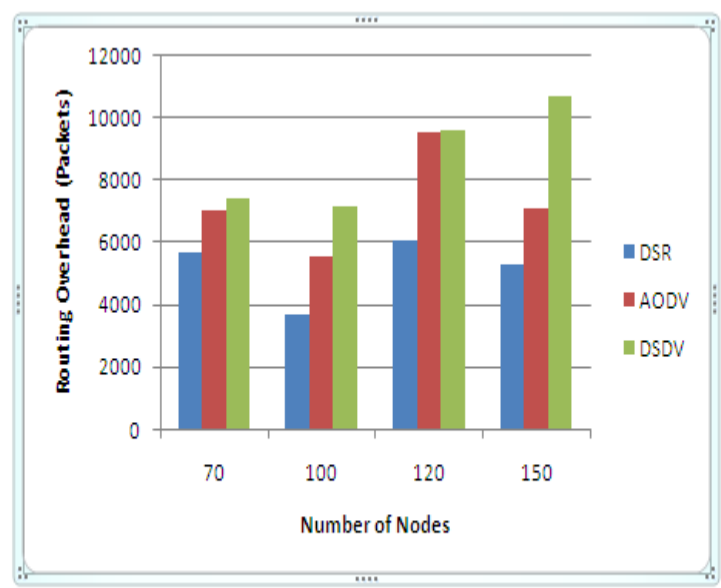

Chart 4.9: Routing overhead as a function of varying nodes

DSDV shows considerable packet loss up to 310 packets at 120 node density whereas the source routing protocols AODV and DSR shows small amount of packet loss, as shown in chart 4.10. None of the protocols show significant change in packet loss except DSDV with the increase in number of nodes.

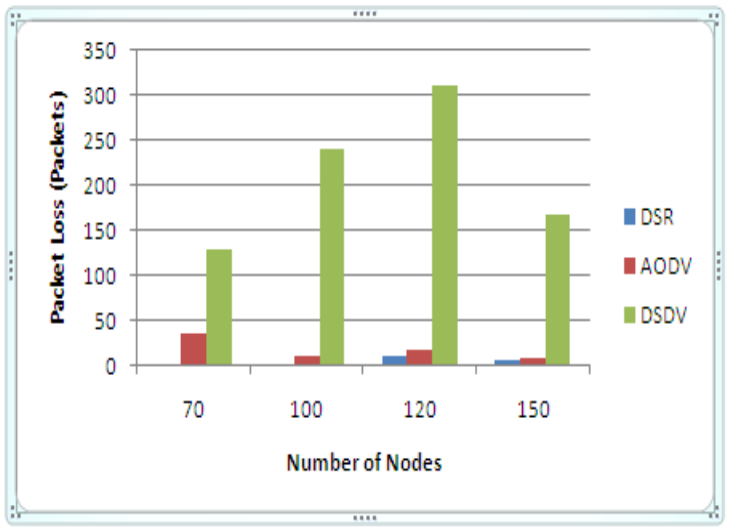

Chart 4.10: Packet loss as a function of varying nodes

\section{CONCLUSIONS}

It is observed that DSDV have a strong effect on their performance as the mobility of the nodes increases so they may not be suitable for the Ad hoc environments with high mobility circumstance. The DSR and AODV performed significantly better than DSDV as they use reactive approach, which eliminates the need to periodically flood the network as compared to table driven approach (proactive approach). DSR has overall good performance even when the mobility is high. Even the performance of DSR is affected with the change in scalability. It is observed that by varying the no. of connections AODV and DSR perform well then DSDV in maximum approaches but high variation is found for AODV in average delay while changing the maximum connections. With the variation of data rate it is observed that DSR and AODV performs well then DSDV, but on sending the packets at data rate of 8 packets/sec. it is found that DSR performance reduces as its varying parameters signifies its negative impact as and if compared with AODV and DSDV. Even loss of packets on this data rate is more as and if compared with other 2 protocols. So we can conclude that there is a need for routing protocols explicitly tuned to the characteristics of ad-hoc networks because the performance of the routing protocols depends on the number of parameters like mobility rate, network size and maximum connections to nodes and data rate.

\section{REFERENCES}

[1] D. Kim, J. Garcia and K. Obraczka, "Routing Mechanisms for Mobile Ad Hoc Networks based on the Energy Drain Rate", IEEE Transactions on Mobile Computing. Vol 2, no 2, 2003, pp.161-173

[2] IETF Working Group: Mobile Ad hoc Networks (MANET). Available at: http://www.ietf.org/html.charters/manetcharter.html.

[3] S.K. Sarkar, T.G. Basawaraju and C Puttamadappa, -Ad hoc Mobile Wireless Networks: Principles, Protocols and Applications, Auerbach Publications, pp. 1, 2008.

[4] C.K. Toh, —Ad Hoc Mobile Wireless Networks: Protocols and Systems, Prentice Hall PTR, 2002.

[5] K. Brown and S. Singh, - M-TCP: TCP for Mobile cellular Networks, Proc. of ACM SIGCOMM Computer Communication Review, pp. 19-43, 1997.

[6] Steve Wisniewski, -Wireless and Cellular networks, Prentice Hall, 2004.

[7] Andrea Goldsmith, -Wireless Communications, Cambridge University Press, 2005.

[8] Bing Lin and I. Chlamtac, - Wireless and Mobile Network Architectures, Wiley, 2000.

[9]Wireless_technologies_advantage_from:http://www.kimaldi. com/kimaldi_eng/area_de_conocimiento/tecnologia_wireless/ ventajas_tecnologia_wireless, 2008.

\section{BIOGRAPHIES}

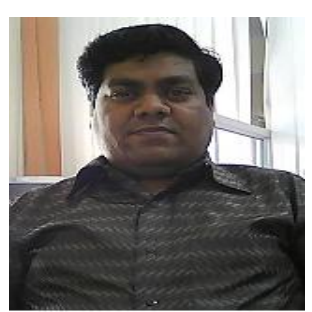

Vishal Shrivastava working as Assistant Professor in Arya College \& IT. He has published many national and international research papers. He has very depth knowledge of his research areas. 